\title{
Topographic mapping of left ventricular regional contractile injury in ischemic mitral regurgitation
}

\author{
Timothy S. Lancaster, MD, Julia Kar, PhD, Brian P. Cupps, PhD, Matthew C. Henn, MD,
}

Kevin Kulshrestha, BS, Danielle J. Koerner, and Michael K. Pasque, MD

\section{ABSTRACT}

Objective: Restrictive leaflet tethering resulting from regional left ventricular (LV) contractile injury causes ischemic mitral regurgitation (MR). We hypothesized that 3-dimensional LV topographic mapping by MRI-based multiparametric strain analysis could characterize the regional contractile injury patterns that differentiate ischemic coronary artery disease patients who have ischemic MR from those who do not.

Methods: Magnetic resonance imaging-based multiparametric strain data were calculated for $15,300 \mathrm{LV}$ grid points in 100 normal volunteers. Strain parameters from ischemic MR $(n=10)$ and ischemic no-MR $(n=36)$ patients were then normalized to this normal human strain database with $z$ score quantification of standard deviation from the normal mean. Mean multiparametric strain $z$ scores were calculated for $18 \mathrm{LV}$ subregions (basilar/mid/apical levels; $6 \mathrm{LV}$ regions). Mean strain $z$ scores for papillary muscle-related (basilar/mid levels of anterolateral, posterolateral, and posterior) and nonpapillary muscle-related (all other) subregions were compared between ischemic MR and ischemic no-MR groups.

Results: Across all patients, contractile injury was greater in the papillary muscle-related regions compared with the nonpapillary regions $(P=.007)$. In the papillary regions, contractile injury was greater in the ischemic MR group compared with the no-MR group $(z$ scores, $1.91 \pm 1.13$ vs $1.20 \pm 1.01$, respectively; $P<.001)$. Strain values in the nonpapillary muscle-related subregions were not different between the 2 groups $(1.31 \pm 1.04 \mathrm{vs}$ $1.20 \pm 1.03 ; P=.301)$.

Conclusions: Multiparametric strain analysis demonstrated severe normalized contractile injury in the papillary muscle-related LV subregions in patients with ischemic MR. The mean degree of normalized injury approached 2 standard deviations and was significantly worse than the levels seen in ischemic no-MR patients. (J Thorac Cardiovasc Surg 2017;154:149-158)

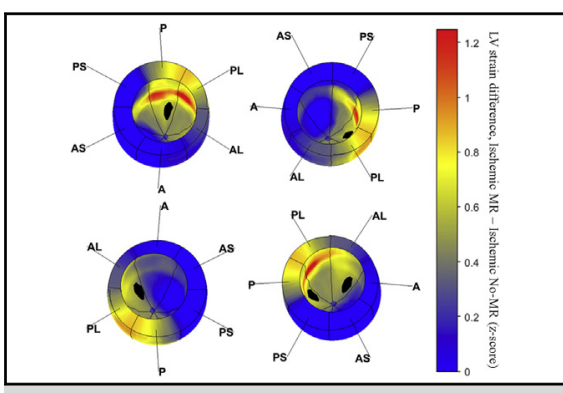

Contractile injury in the papillary regions distinguishes patients with ischemic mitral regurgitation from those without mitral regurgitation.

Central Message

MRI-based 3D topographic mapping of regional LV contractile injury patterns differentiates patients with ischemic MR from patients without ischemic MR.

\section{Perspective}

Because mitral leaflet tethering from regional left ventricular contractile injury is the root cause of ischemic mitral regurgitation, characterization of associated contractile injury distribution may affect management. The imaging and analytical capabilities necessary to perform a high-resolution topographic 3-dimensional mapping of regional contractile injury have only recently become clinically available.

See Editorial Commentary page 159

\footnotetext{
From the Division of Cardiothoracic Surgery, Washington University School of Medicine, Barnes-Jewish Hospital, St Louis, Mo.

Supported in part by funding from the National Institutes of Health grants HL064869, HL069967, HL112804, and T32 HL007776.

Read at the 96th Annual Meeting of The American Association for Thoracic Surgery, May 14-18, 2016, Baltimore, Maryland.

Received for publication May 27, 2016; revisions received Oct 28, 2016; accepted for publication Nov 1, 2016; available ahead of print Jan 18, 2017.

Address for reprints: Michael K. Pasque, MD, Washington University School of Medicine, 660 S Euclid Ave, Campus Box 8234, St Louis, MO 63110 (E-mail: pasquem@wudosis.wustl.edu).

$0022-5223 / \$ 36.00$

Copyright (c) 2016 by The American Association for Thoracic Surgery

http://dx.doi.org/10.1016/j.jtcvs.2016.11.055
}

Ischemic mitral regurgitation (MR) is characterized by a structurally normal mitral valve (MV) with regurgitation due to left ventricular (LV) dysfunction. ${ }^{1}$ The primary root cause of

Scanning this QR code will take you to the supplemental videos and table for the article. To view the AATS 2016 Webcast, see the URL next to the video thumbnail.

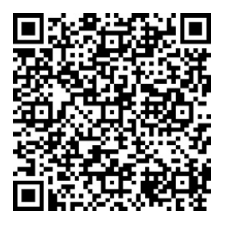




$$
\begin{aligned}
& \text { Abbreviations and Acronyms } \\
& \begin{aligned}
\text { 3D } & =3 \text {-dimensional } \\
\mathrm{CAD} & =\text { coronary artery disease } \\
\mathrm{LV} & =\text { left ventricle } \\
\mathrm{MR} & =\text { mitral regurgitation } \\
\mathrm{MRI} & =\text { magnetic resonance imaging } \\
\mathrm{MV} & =\text { mitral valve }
\end{aligned}
\end{aligned}
$$

ischemic MR is restrictive leaflet tethering resulting from regional LV ischemic contractile injury. ${ }^{2}$ Displacement of the papillary muscles, especially the posteromedial papillary muscle, leads to restrictive tethering and deformation of the MV leaflets with impaired leaflet coaptation and resulting regurgitation. ${ }^{3,4}$ The regional distribution of the ischemic contractile injury is key in determining the occurrence, severity, and exact mechanism of subsequent $\mathrm{MR}$ in patients with coronary artery disease (CAD) and myocardial infarction. ${ }^{1,5}$

Until recently, the standard of care in the surgical treatment of ischemic MR has been revascularization combined with undersized mitral ring annuloplasty. Unfortunately, recent randomized trial data have demonstrated recurrence of at least moderate MR in up to $59 \%$ of patients undergoing MV repair by 2 years of follow-up, in comparison to only $4 \%$ of patients undergoing MV replacement. ${ }^{6,7}$ Although these results suggest that the long-term outcomes of MV replacement may be superior to MV repair, the higher perioperative mortality, sacrifice of the native valve apparatus, potential long-term prosthesis deterioration, and need for anticoagulation associated with MV replacement cannot be overlooked. ${ }^{2,8-10}$ Given the considerable disadvantages of MV replacement, the remaining $40 \%$ of patients who may do well with MV repair should not be abandoned to valve replacement for a lack of clinically applicable prognostic metrics.

Because recurrent or persistent MR after annuloplasty repair is likely to occur by the same mechanism as primary ischemic MR (ie, leaflet tethering by ischemia-induced regional contractile injury and remodeling), regional contractile injury distribution patterns have potential to predict repair failure in ischemic MR. Whereas several echocardiographic features have been shown to correlate with MR recurrence after MV repair, including measures of leaflet and chordal geometry ${ }^{11-14}$ and measures of LV remodeling, ${ }^{15-18}$ these are all indirect surrogates of the regional ventricular contractile dysfunction responsible for ischemic MR. Logically, our first goal must be the full characterization of the ischemia-induced contractile injury distribution patterns that are associated with preoperative ischemic MR, because variants of these prognostic patterns have the highest likelihood of providing metrics to predict recurrent or persistent MR after repair.

Although speckle-tracking echocardiography can supply some degree of quantitative measurements of global and regional contractile function, cardiac MRI is widely regarded as the most accurate imaging modality for quantitative assessment of LV function. ${ }^{19,20}$ A high-resolution, highly quantitative characterization of regional contractile function is necessary to uncover the complexities of the contractile injury distribution that causes ischemic MR. Only recently has a quantitative description of truly 3-dimensional (3D) regional LV contractile function by regional $3 \mathrm{D}$ strain calculation from MRI-derived LV displacement data become clinically available. We have demonstrated the high reliability of MRI-based multiparametric strain topographic mapping in the quantification and localization of regional $\mathrm{LV}$ contractile injury in patients with $\mathrm{CAD} .{ }^{21}$ As a first step in evaluating the clinical application of this technology to ischemic MV disease, we sought to fully map the associated distribution patterns of regional contractile injury in patients with ischemic MR. We hypothesized that topographic strain mapping of regional LV contractile function could characterize the regional contractile injury patterns that differentiate ischemic CAD patients who have MR from those who do not.

\section{METHODS}

\section{Study Participants}

The Institutional Review Board of Washington University School of Medicine approved this study, and all subjects gave written informed consent. One hundred forty-six total study participants were enrolled in the study and underwent cardiac MRI with radiofrequency tissue tagging. One hundred of these participants were normal, healthy volunteers with no historical, physical, or clinical evidence of heart disease who contributed complete strain parameter information to a normal human strain database. The remaining 46 participants were patients with ischemic CAD, 10 of whom had ischemic MR and 36 of whom had ischemic CAD without MR. CAD was defined as $\geq 1$ lesion of $\geq 70 \%$ stenosis on coronary angiography as interpreted by an independent cardiac catheterization laboratory blinded to study results. MR was defined as moderate $(\geq 2)$ or greater MR as determined by an independent echocardiography laboratory blinded to study results. Restricted leaflet motion was the cause of the MR in all 10 patients with ischemic MR.

\section{Cardiac MRI}

All imaging studies were carried out using a $1.5 \mathrm{~T}$ scanner (Siemens, Erlangen, Germany). Short-axis grid-tagged MR images were acquired in a stack starting at the level of the MV and extending to the apex of the LV, whereas long-axis line-tagged images were acquired in 4 radially oriented planes intersecting at the approximate center of the LV cavity (Figure 1, A). In each imaging plane, a spatial modulation of magnetization radiofrequency tissue-tagging preparation was applied, followed by a 2-dimensional balanced steady-state free precession cine image acquisition. ${ }^{22,23}$ This process creates markers on the myocardium that deform with the tissue, which allows for quantification of wall motion. Typical imaging parameters were repetition time $32.4 \mathrm{~ms}$, echo time $1.52 \mathrm{~ms}$, field of view $350 \times 350 \mathrm{~mm}$, flip angle $20^{\circ}$, tag spacing $8 \mathrm{~mm}$, and slice thickness $8 \mathrm{~mm}$.

\section{Strain Analysis}

Strain measurements were obtained for all study participants using an established and validated method developed at our institution. ${ }^{24-27}$ Clinical validation of this methodology has demonstrated resistance to interobserver variability. ${ }^{28,29}$ Endocardial and epicardial wall boundaries were manually identified on each of the images. Tag lines were tracked 


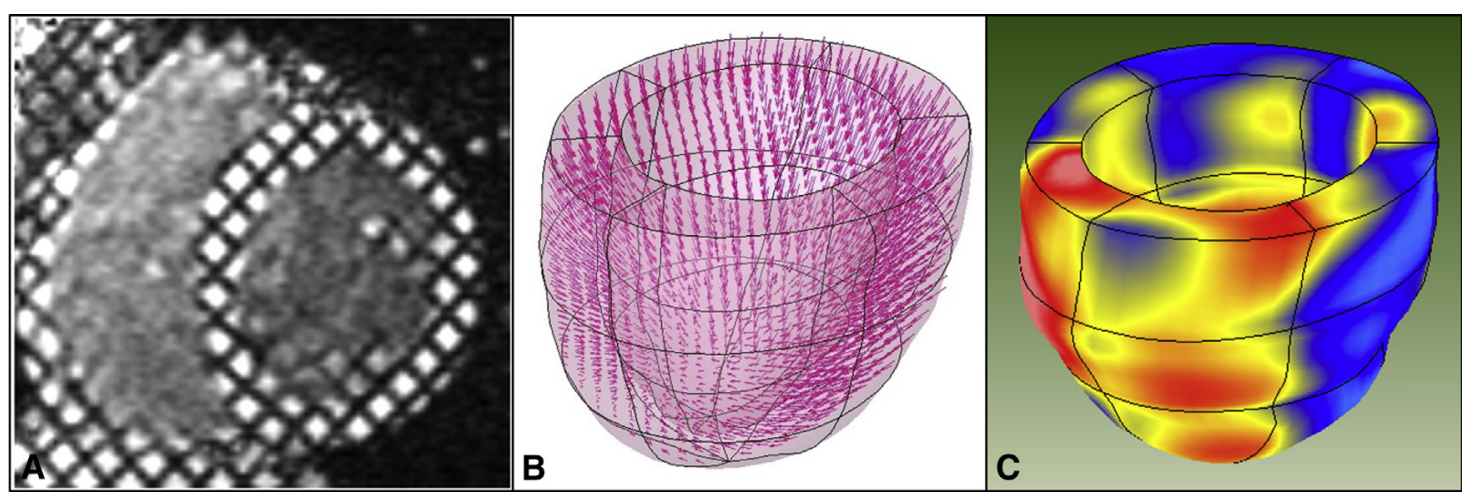

FIGURE 1. The steps involved in obtaining the strain measurements in this study. A, A representative short-axis magnetic resonance imaging (MRI) tagged image. B, A standardized 18-region finite element model showing MRI measured displacements. C, A color contour plot showing multiparametric $z$ scores obtained by comparison to the normal strain database.

on each of the images semiautomatically utilizing an active contour approach. $^{24}$

3D displacements were computed for systole from the intramyocardial intersection points of the 3 tag surfaces. The end-diastolic wall boundaries were used to create a finite element model of the LV. Using the anterior and posterior insertion points of the right ventricle to the LV as landmarks, a standardized 18-element mesh was created for each model (Figure 1, B). A least squares fitting of the displacement data provided the strain measurements for this study. Circumferential strain and longitudinal strain were computed over a standardized grid of 15,300 points for the entire LV (Table E1). The finite element software package StressCheck (ESRD, Inc, St Louis, Mo) was used to obtain the strain measurements for this study.

\section{Normalization and Multiparametric Strain Analysis}

A normal human strain database was constructed using strain measurements from the 100 normal participants. At each LV grid point and for each strain parameter, measurements from the normal participants were used to generate a normal mean and standard deviation (SD) at that particular point. This database of normal strain values allows for patientspecific, point-specific, and parameter-specific comparisons with $z$ score generation. A $z$ score is a statistical measurement that relates an individual value to the mean of a group of values, and represents the number of SDs by which the patient strain parameter value varies from the normal mean value. For example, if a patient's circumferential strain parameter has a $z$ score of 1.57 at a particular point, this means that his or her contractile function in the area of that grid point is 1.57 SDs worse than the normal average.

For the 46 test patients with CAD with or without MR, $z$ scores for both circumferential and longitudinal strain were calculated at every LV grid point by reference to the normal human strain database. The $z$ scores for the 2 strain parameters were then averaged to generate a single multiparametric strain $z$ score at each point. Because 2 strain parameters were normalized at each of 15,300 LV grid points for each patient study, each patient's LV color contour topographic mapping of multiparametric strain values represents a total of 30,600 individual database comparisons (Figure $1, C$ ). Worse contractile function results in more positive $z$ scores.

\section{Subregional LV Strain Analysis}

Mean multiparametric strain $z$ scores were calculated for the $18 \mathrm{LV}$ subregions, which included the basilar, mid, and apical levels of the anterior, anterolateral, posterolateral, posterior, posteroseptal, and anteroseptal regions. Subregions were classified as either papillary muscle-related (basilar and mid levels of anterolateral, posterolateral, and posterior) and nonpapillary muscle-related (all other subregions).
Mean multiparametric strain $z$ scores for the papillary and nonpapillary muscle subregions were compared between the ischemic MR and ischemic no-MR groups.

\section{Statistical Analysis}

Continuous variables were expressed as means \pm SD and were compared using 2-sample $t$ tests. Categorical variables were expressed as frequencies and percentages, and were compared using $\chi^{2}$ analysis. Average multiparametric strain $z$ scores in the papillary and nonpapillary muscle-related subregions were compared between the ischemic MR and ischemic no-MR groups using a mixed analysis of variance analysis, with post hoc analysis for between-group effects by 2 -sample $t$ tests. The Shapiro-Wilks test was used to assess the normality of the data, and Levene's test was used to test the equality of error variances. Data analyses were performed using SPSS 23.0 statistical software (IBM-SPSS Inc, Armonk, NY). Composite 3D LV color contour multiparametric strain

TABLE 1. Demographic and clinical characteristics of study test patients with and without mitral regurgitation (MR)

\begin{tabular}{lccc}
\hline & $\begin{array}{c}\text { Ischemic } \\
\text { MR }\end{array}$ & $\begin{array}{c}\text { Ischemic } \\
\text { No-MR }\end{array}$ & $\begin{array}{c}\boldsymbol{P} \\
\text { value }\end{array}$ \\
\hline $\mathrm{N}$ & 10 & 36 & \\
$\mathrm{Age}, \mathrm{y}$ & $61.6 \pm 8.7$ & $61.8 \pm 13.3$ & .947 \\
Female & $40(4 / 10)$ & $19(7 / 36)$ & .220 \\
Hypertension & $100(8 / 8)$ & $81(29 / 36)$ & .318 \\
Diabetes & $38(3 / 8)$ & $22(8 / 36)$ & .391 \\
Chronic obstructive pulmonary disease & $0(0 / 8)$ & $8(3 / 36)$ & 1.0 \\
Chronic renal insufficiency & $50(4 / 8)$ & $31(11 / 36)$ & .414 \\
Atrial fibrillation & $13(1 / 8)$ & $17(6 / 36)$ & 1.0 \\
New York Heart Association & $25(2 / 8)$ & $61(22 / 36)$ & .115 \\
$\quad$ functional class I & & & \\
New York Heart Association & $63(5 / 8)$ & $28(10 / 36)$ & .099 \\
$\quad$ functional class II & & & \\
New York Heart Association & $13(1 / 8)$ & $11(4 / 36)$ & 1.0 \\
$\quad$ functional class III & & & \\
History of myocardial infarction & $60(6 / 10)$ & $61(22 / 36)$ & 1.0 \\
Previous coronary artery bypass graft & $30(3 / 10)$ & $33(12 / 36)$ & 1.0 \\
Previous percutaneous coronary & $50(5 / 10)$ & $47(17 / 36)$ & 1.0 \\
$\quad$ intervention & & & \\
\hline Values are presented as n, mean \pm standard deviation, or \% (n/N). &
\end{tabular}


maps were created using Matlab computing software (MathWorks, Inc, Natick, Mass).

\section{RESULTS}

Clinical characteristics of the study test patients are shown in Table 1 and echocardiographic and cardiac catheterization characteristics are shown in Table 2. There were no significant differences between the ischemic MR and ischemic no-MR patient groups in the prevalence of evaluated medical comorbidities, the distribution of coronary artery lesions, or echocardiographic evaluation of LV ejection fraction or regional ventricular dysfunction.

The analysis of variance revealed a significant main effect for the LV subregion $(F[1,44]=7.877 ; P=.007$; observed power $=0.784$ ), indicating that $z$ scores in the papillary regions were greater than those in the nonpapillary regions without regard to the presence of MR. This analysis also indicated a significant interaction between the presence of MR and the LV subregion $(F[1,44]=6.588 ; P=.014$, observed power $=0.706$ ). On subregional analysis, strain values for the papillary muscle-related subregions were significantly higher in the ischemic MR group compared with ischemic no-MR group $(1.91 \pm 1.13$ vs $1.20 \pm 1.01$; $P<.001$ ) (Figure 2). In contrast, strain values in the nonpapillary muscle-related subregions were not different between the ischemic MR and ischemic no-MR groups

TABLE 2. Echocardiographic and cardiac catheterization characteristics of study test patients with or without mitral regurgitation (MR)

\begin{tabular}{lccc}
\hline & $\begin{array}{c}\text { Ischemic } \\
\text { MR }\end{array}$ & $\begin{array}{c}\text { Ischemic } \\
\text { No-MR }\end{array}$ & $\begin{array}{c}\boldsymbol{P} \\
\text { value }\end{array}$ \\
\hline $\mathrm{N}$ & 10 & 36 & \\
MR grade: & & & \\
$\quad$ None & $0(0 / 10)$ & $56(20 / 36)$ & .002 \\
$\quad$ Mild (1+) & $0(0 / 10)$ & $44(16 / 36)$ & .009 \\
$\quad$ Moderate (2+) & $50(5 / 10)$ & $0(0 / 36)$ & $<.001$ \\
$\quad$ Severe (3+) & $50(5 / 10)$ & $0(0 / 36)$ & $<.001$ \\
Active coronary artery disease* & $70(7 / 10)$ & $67(24 / 36)$ & 1.0 \\
No. of diseased vessels & $2.5 \pm 0.7$ & $2.2 \pm 0.8$ & .187 \\
$\quad$ Left anterior descending artery & $100 \%(10 / 10)$ & $94(34 / 36)$ & 1.0 \\
Circumflex artery & $60(6 / 10)$ & $83(30 / 36)$ & .190 \\
$\quad$ Right coronary artery & $60(6 / 10)$ & $75(27 / 36)$ & .435 \\
LV ejection fraction, \% & $43.1 \pm 12.9$ & $43.6 \pm 11.9$ & .920 \\
LV end diastolic diameter, cm & $5.3 \pm 0.7$ & $5.5 \pm 1.2$ & .618 \\
LV end systolic diameter, cm & $4.1 \pm 0.8$ & $4.1 \pm 0.9$ & .879 \\
LV wall thickness, cm & $1.1 \pm 0.1$ & $1.2 \pm 0.2$ & .230 \\
Regional wall dyskinesis & & & \\
$\quad$ Anterior & $40(4 / 10)$ & $50(18 / 36)$ & .725 \\
Septum & $40(4 / 10)$ & $44(16 / 36)$ & 1.0 \\
Lateral & $40(4 / 10)$ & $44(16 / 36)$ & 1.0 \\
Posterior & $20(2 / 10)$ & $22(8 / 36)$ & 1.0 \\
Inferior & $50(5 / 10)$ & $42(15 / 36)$ & .726 \\
\hline Valesare & &
\end{tabular}

Values are presented as $\mathrm{n}, \%(\mathrm{n} / \mathrm{N})$, or mean \pm standard deviation. $L V$, Left ventricular. *Symptomatic.
$(1.31 \pm 1.04$ vs $1.20 \pm 1.03 ; P=.301)$ (Figure 2). Global $z$ scores were not found to be significantly different between the MR and no MR groups $(F[1,44]=2.049 ; P=.159)$.

The availability of multiparametric strain data at each of 15,300 points in a uniform, standardized LV grid for every patient in each of the 2 groups enables a unique visual presentation of each group's mean regional strain data. Composite 3D LV color contour maps displaying the topographic distribution of the average, normalized multiparametric strain for each group are shown in Figure 3. Each color map demonstrates the regional normalized contractile injury distribution of an average patient with ischemic no-MR (Figure 3, $A$ ) or with ischemic MR (Figure 3, B). The images represent 4-quadrant endocardial views from each group's composite, highresolution, rotatable 3D LV model. Full videos of the rotating contour maps are available in Video 1. The group composite maps highlight the differences in regional contractile injury distribution between the ischemic no-MR and ischemic MR groups, and clearly illustrate a greater degree of contractile injury in the papillary muscle-related subregions for ischemic MR patients.

Further, because both study groups have average $z$ score values for each strain parameter at each $\mathrm{LV}$ grid point, this methodology also uniquely allows an intuitive visual display of the differences in normalized regional contractile injury distribution, obtained by subtracting the 15,300 point-specific average $z$ scores between the 2 patient subgroups. Thus, by simply subtracting the average multiparametric strain metric $z$ scores at each LV grid point, we can see, in the resulting composite subtraction map, the precise contractile injury distribution that causes ischemic MR. The composite 3D LV subtraction map is shown in Figure 4 and in Video 2. The composite subtraction map highlights the more severe normalized contractile injury in the ischemic MR group and the localization of this difference to the papillary muscle-related subregions, most notably in the region of the posteromedial papillary muscle. A lesser but notable difference in normalized contractile injury was associated with the anterolateral papillary muscle. In the red regions of the 3D LV map, the ischemic MR group demonstrates $>1 \mathrm{SD}$ worse contractile injury than the ischemic no-MR group. The blue color represents regions in which there is no difference in contractile function between the 2 groups.

\section{DISCUSSION}

Ischemic $M R$ is caused by regional ventricular contractile injury-based papillary muscle displacement resulting in restrictive leaflet tethering. ${ }^{1}$ Leaflet tethering, changes in chordal geometry, and all of the other structural metrics that have been associated with ischemic MR occur as a consequence of regional contractile abnormalities and are therefore secondary surrogates of the contractile injury 


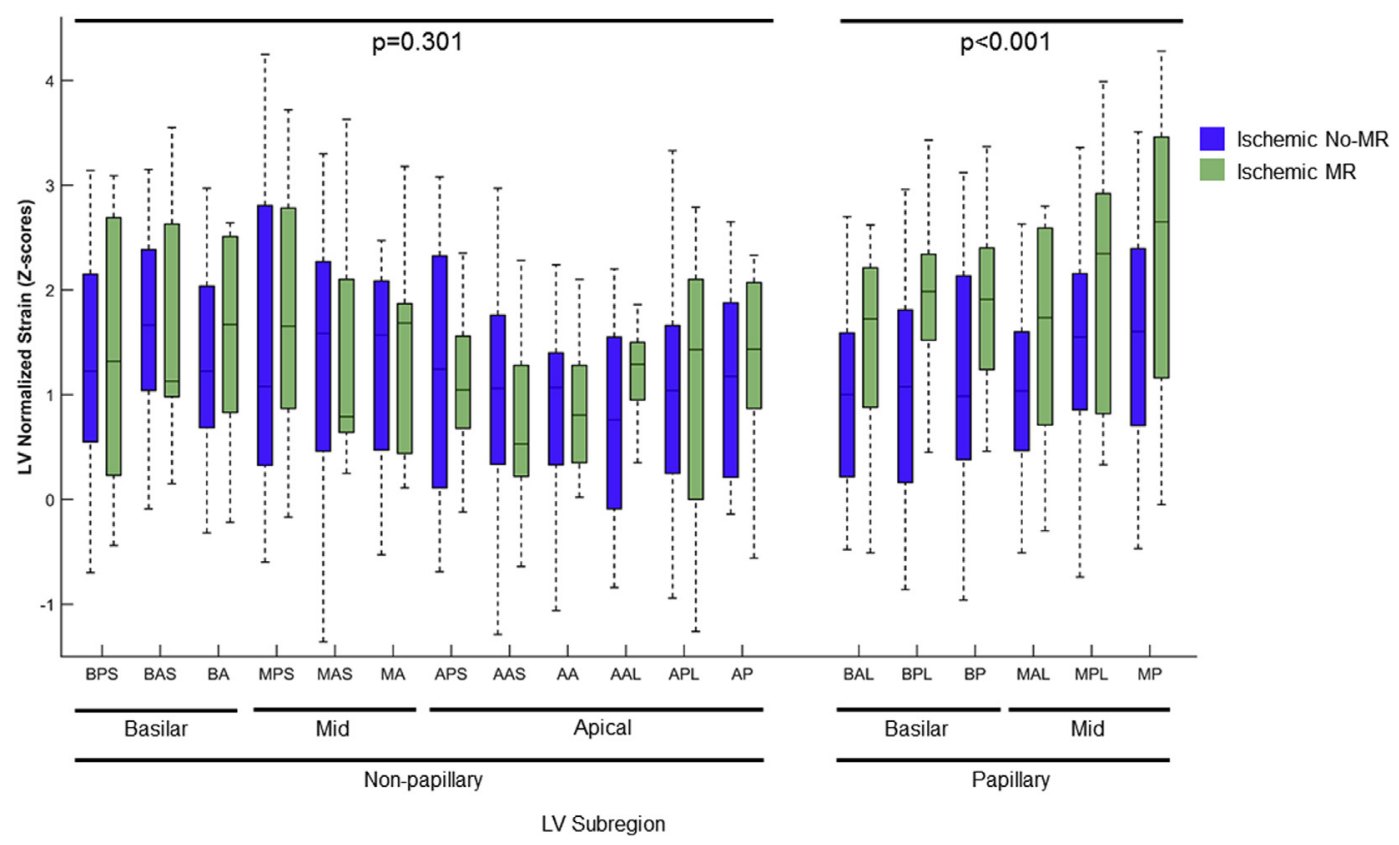

FIGURE 2. Box plots of normalized multiparametric strain $z$ score values by left ventricular $(L V)$ subregion for ischemic no mitral regurgitation ( $M R$ ) versus ischemic MR patient groups, organized by nonpapillary muscle-related subregions (left) and papillary muscle-related subregions (right). Boxes represent 25 th to 75 th percentile, whereas error bars represent minimum and maximum values in the dataset. $P$ values represent ischemic no-MR versus ischemic MR comparison within the nonpapillary and papillary subregion groups. BPS, Basilar posteroseptal; BAS, basilar anteroseptal; BA, basilar anterior; $M P S$, mid posteroseptal; $M A S$, mid anteroseptal; $M A$, mid anterior; $A P S$, apical posteroseptal; $A A S$, apical anteroseptal; $A A$, apical anterior; $A A L$, apical anterolateral; $A P L$, apical posterolateral; $A P$, apical posterior; $B A L$, basilar anterolateral; $B P L$, basilar posterolateral; $B P$, basilar posterior; $M A L$, midanterolateral; $M P L$, midposterolateral; $M P$, midposterior.

demonstrated in Figures 2-4. The most likely source for clinically useful metrics that can predict the occurrence of MR in ischemic CAD patients is this primary root cause: regional contractile injury.
This study used MRI-based multiparametric strain analysis to topographically map normalized regional LV contractile injury in ischemic CAD patients with and without MR. Multiparametric strain analysis provides an
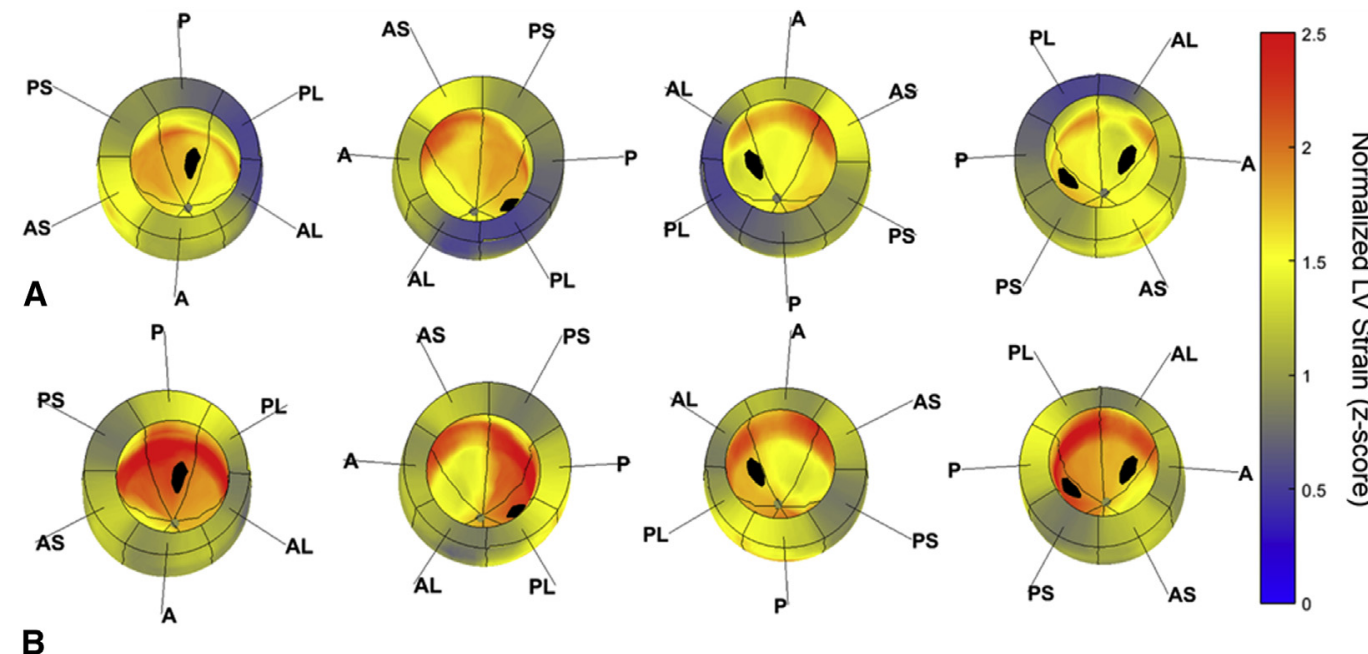

в

FIGURE 3. By averaging multiparametric strain $z$ scores in all patients in each group at every left ventricular $(L V)$ grid point, a topographic map of average regional normalized contractile injury distribution for each group is uniquely visualized. These images represent the normalized contractile injury distribution of an average patient from each group. Shown are 4-quadrant endocardial views of the composite 3-dimensional LV color contour maps of the ischemic no mitral regurgitation group (A) and ischemic mitral regurgitation group (B). The color scale represents a 2.5 standard deviation range. Black ovals represent approximate papillary muscle insertion sites. $P S$, Posteroseptal; $P$, posterior; $P L$, posterolateral; $A$, anterior; $A L$, anterolateral; $A S$, anteroseptal. 

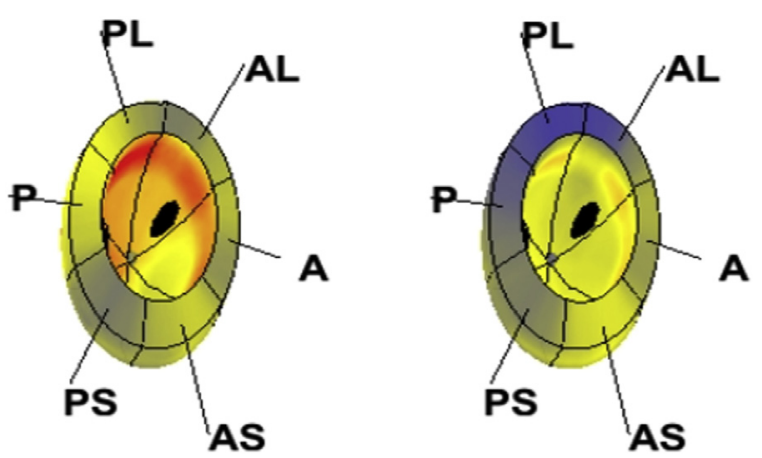

VIDEO 1. By averaging multiparametric strain $z$ scores in all patients in each group at every left ventricle (LV) grid point, a 3-dimensional (3D), rotatable topographic LV map of average regional normalized contractile injury distribution for each group can be uniquely visualized. This video shows rotating views of the 2 group maps representing the normalized contractile injury distribution of an average patient from each group. Shown here are rotating composite 3D LV color contour maps of the ischemic mitral regurgitation (left) and ischemic no mitral regurgitation (right) groups. The color scale represents a 2.5 standard deviation range. Black ovals represent approximate papillary muscle insertion sites. $P L$, Posterolateral; $A L$, anterolateral; $A$, anterior; $A S$, anteroseptal; $P S$, posteroseptal; $P$, posterior. Video available at: http://www. jtcvsonline.org/article/S0022-5223(16)31679-8/addons.

objective, highly quantified measure of myocardial contractile function and viability. ${ }^{21,30,31}$ Higher multiparametric strain $z$ score values are reflective of worse contractile function, with strain $z$ scores $>1.5$ accurately identifying regions of nonviable myocardium. ${ }^{31}$ We have recently demonstrated the reliability of this technology in comparison to other gold standard clinical metrics for evaluating the heterogeneity of ventricular dysfunction in patients with CAD and previous infarction. ${ }^{21}$

In this study, multiparametric strain analysis demonstrated worse global contractile function in the ischemic MR group. This difference in contractile function was primarily localized to the papillary muscle-related LV subregions, because the ischemic MR group had markedly worse contractile injury in the basilar and mid levels of the anterolateral, posterolateral, and posterior subregions. Average strain $z$ scores in the region of the posteromedial papillary muscle were almost 2 SDs worse than normal in the ischemic MR group (Figure 3), and exceeded the ischemic no-MR group by $>1$ SD (Figure 4). This is a very large difference and demonstrates the severity of the contractile injury localized to these regions in the ischemia patients who manifest MR.

Multiparametric strain analysis of regional LV contractile function was therefore able to characterize the regional contractile injury patterns that differentiate ischemic CAD patients who have MR from those who do not. A specific and discernible topographic pattern of regional LV contractile injury is seen in patients with ischemic MR that is easily differentiated from the injury pattern seen in ischemic patients with no MR. An advantage of presenting the group average subtraction data in a visual format is the ability to visually associate the profound group subtraction differences in normalized regional contractile injury with the 2 mitral papillary muscle insertion sites (Figure 4). Ventricular function in the nonpapillary muscle-related subregions was not different between the ischemic MR and ischemic no-MR groups. These findings are consistent with the known pathophysiology of MR, in that regional ventricular dysfunction affecting the papillary muscles leads to tethering, deformation, and insufficiency of the valve leaflets. ${ }^{1}$

Although these findings support the potential clinical applicability of this technology in the detailed characterization of ventricular function in patients with ischemic CAD and resulting MV disease, this study is admittedly a step removed from predicting MR recurrence after MV repair. Given that nearly $60 \%$ of patients undergoing MV repair for ischemic MR experience recurrence by 2 years of follow-up, ${ }^{7}$ reliable metrics for predicting repair failure are needed. Multiple echocardiographic predictors of repair failure have been proposed ${ }^{11-18}$; however, in a recent follow-up analysis to the Cardiothoracic Surgical Trials Network study on severe ischemic MR, no measures of MV leaflet tethering or geometry were significantly associated with MR recurrence. The presence of basal aneurysm or dyskinesis was strongly associated with recurrent MR and was included in a pilot multivariable predictive model with moderate performance for prediction of recurrent MR. ${ }^{18}$

The potential for reverse remodeling and recovery of regional ventricular function is a key determinant of the success of MV repair, as illustrated by the interesting finding that greater LV reverse remodeling occurs in patients who undergo MV repair and do not experience recurrence, compared with patients undergoing MV replacement. $^{32}$ In patients with positive LV reverse remodeling after MV repair for ischemic MR (reduction in LV end-systolic volume index $\geq 15 \%$ ), freedom from recurrent MR was $>90 \% .{ }^{33}$ Similarly, patients in the Cardiothoracic Surgical Trials Network trial who underwent MV repair without recurrence had lower LV end-systolic volume indices at follow-up than those who experienced recurrence. ${ }^{7,10}$ These data suggest that MV repair is more likely to be successful in patients with potential for reverse ventricular remodeling.

The ability to objectively and reliably identify patients with potential for regional LV contractile recovery, especially in the papillary muscle-related regions, is a unique strength of MRI-based multiparametric strain analysis and may provide insight into the decision regarding 

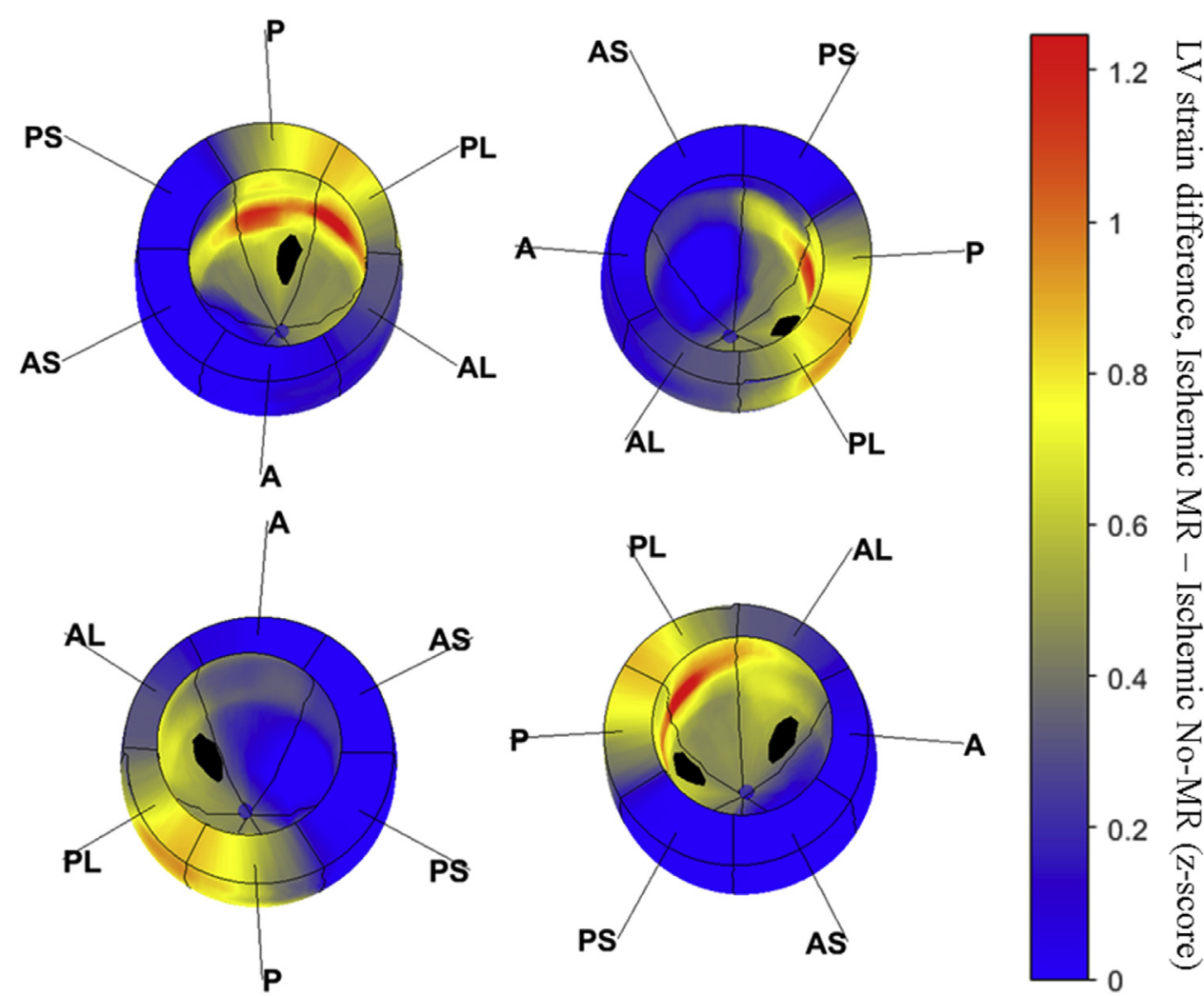

FIGURE 4. The overarching goal of this study was to visually demonstrate the differences in normalized contractile injury topographic distribution between those patients with coronary artery disease who have ischemic mitral regurgitation $(M R)$ and those who do not. Shown here are 4-quadrant endocardial views of a subtraction 3-dimensional left ventricular $(L V)$ color contour map demonstrating differences in LV strain distribution between ischemic MR and ischemic no-MR groups. This unique visualization is only possible because of the availability of group average multiparametric strain $z$ score values at each of 15,300 points in a uniform, standardized grid in both patient groups, which can therefore be subtracted from each other. These 4 views of this composite LV mapping of the subtraction differences between the groups demonstrate the normalized contractile injury distribution that is responsible for the occurrence of MR in this ischemic coronary artery disease patient population. The color scale represents a 1.2 standard deviation range in subtraction differences between the 2 patient groups. Red regions represent $>1$ full standard deviation increase in contractile injury in the ischemic MR group, whereas blue areas represent no difference between the 2 groups. Black ovals represent approximate papillary muscle insertion sites. $P S$, Posteroseptal; $P$, posterior; $P L$, posterolateral; $A$, anterior; $A L$, anterolateral; $A S$, anteroseptal.

MV repair versus replacement. Our previous work has demonstrated the ability of this technology to reliably characterize regional LV myocardial viability in ischemic cardiomyopathy ${ }^{31}$ and to predict regional contractile recovery after aortic valve replacement. ${ }^{34}$ In previous studies we have demonstrated that a multiparametric strain $z$ score regional average $>1.52$ is highly associated with irreversible regional myocardial injury, detecting nonviability with $>90 \%$ sensitivity and specificity. ${ }^{31}$ Minimal contractile recovery potential is expected in subregions demonstrating such severe impairment of normalized contractile function. We are particularly encouraged that this contractile metric may have the potential to uncover the complex topographic injury distribution patterns that correlate with repair failure in ischemic MR, which is the focus of ongoing work in our laboratory.

Limitations of the proposed clinical application of MRI-based multiparametric stain analysis include the expense of MRI and the time requirement for postprocessing of myocardial strain data. However, with advances in MR image acquisition technology and moreautomated data analysis, our current scan times are $<30$ minutes, with associated data analysis requiring $<20$ minutes. Another potential limitation of this methodology, common to other measurements of regional myocardial contractility, is that it can quantify only myocardial strain. Ventricular contractility is embodied in strain (ie, how well the wall is moving), stress (ie, how much force the wall is exerting), and time (ie, how quickly the wall is moving). Ideally, stress and strain would be concurrently quantified. Unfortunately, current methods for accurately quantifying regional myocardial stress in the clinical setting are severely limited.

\section{CONCLUSIONS}

The highly quantified characterization of LV contractile injury distribution patterns by MRI-based multiparametric strain analysis demonstrated clear and significant 


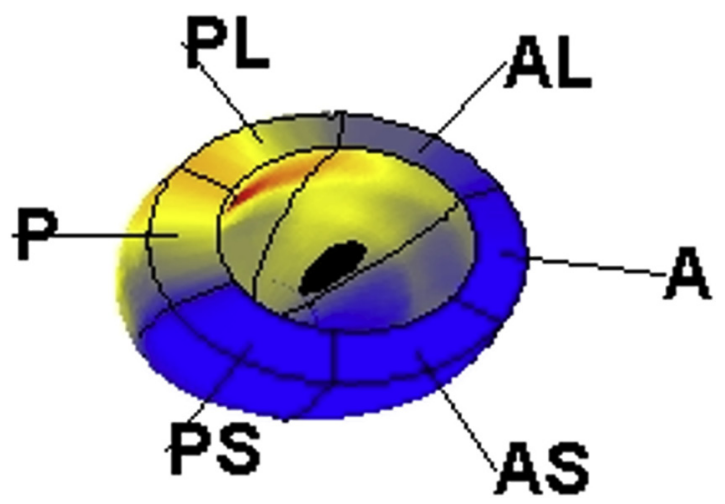

VIDEO 2. The overarching goal of this study was to visually demonstrate the topographic differences in normalized contractile injury distribution between those ischemic coronary artery disease patients who have ischemic mitral regurgitation (MR) and those who do not. Shown here is a video of a rotating subtraction 3-dimensonal left ventricle (LV) color contour map demonstrating differences in LV strain distribution between ischemic MR and ischemic no-MR groups. This unique visualization is only possible because of the availability of group average multiparametric strain $z$ score values at each of 15,300 points in a uniform, standardized grid in both patient groups (with and without ischemic MR), which can therefore be subtracted from each other. This rotating composite LV map of the subtraction differences between the groups demonstrates the normalized contractile injury distribution that is responsible for the occurrence of MR in this ischemic patient population. The color scale represents a 1.2 standard deviation range in subtraction differences between the 2 patient groups. Red regions represent $>1$ full standard deviation increase in contractile injury in the ischemic MR group, whereas blue areas represent no difference between the 2 groups. Black ovals represent approximate papillary muscle insertion sites. $A L$, Anterolateral; $A$, anterior; $A S$, anteroseptal; $P S$, posteroseptal; $P$, posterior; $P L$, posterolateral. Video available at: http://www.jtcvsonline.org/article/ S0022-5223(16)31679-8/addons.

differences between those ischemic CAD patients who have ischemic MR and those who do not. Further, this methodology has unique capabilities in regard to the intuitive presentation of study group summary data in 3D, rotatable, LV color contour, normalized, multiparametric strain maps. Because all regional contractile function is already normalized to a population of healthy volunteers (ie, normal human strain database), the interpretation of summated raw strain data results is provided. Specifically, color contouring based on regional SDs from the normal mean clearly delineates severely injured regions on easily interpreted 3D LV maps. These maps additionally allow a visual summation of the results of this investigation by the simple subtraction of the 2 study group maps. This subtraction color contoured LV map demonstrates the summated differences between those ischemic CAD patients who have ischemic MR and those who do not. This methodology uniquely allows an intuitive visual demonstration of the overarching goal of this investigation that is not available with any other clinically applicable modality: A color contour LV subtraction map representing the normalized regional contractile injury distribution pattern in ischemic CAD patients that results in ischemic MR.

\section{Webcast}

You can watch a Webcast of this AATS meeting presentation by going to: http://webcast.aats.org/2016/Video/Tuesday/ 05-17-16_Ballroom_IV_0800_Lancaster-800.mp4.

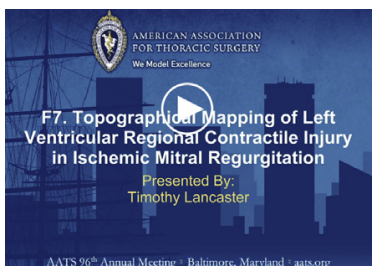

\section{Conflict of Interest Statement}

M.K.P. and B.P.C. may receive royalty income based on a technology developed by them and licensed by Washington University to Cardiowise, Inc. That technology is used in this research. All other authors have nothing to disclose with regard to commercial support.

\section{References}

1. Kron IL, Acker MA, Adams DH, Ailawadi G, Bolling SF, Hung JW, et al. 2015 The American Association for Thoracic Surgery Consensus Guidelines: Ischemic mitral valve regurgitation. J Thorac Cardiovasc Surg. 2016;151:940-56.

2. Di Salvo TG, Acker MA, Dec GW, Byrne JG. Mitral valve surgery in advanced heart failure. J Am Coll Cardiol. 2010;55:271-82.

3. Dagum P, Timek TA, Green GR, Lai D, Daughters GT, Liang DH, et al. Coordinate-free analysis of mitral valve dynamics in normal and ischemic hearts. Circulation. 2000;102:III62-9.

4. Godley RW, Wann LS, Rogers EW, Feigenbaum H, Weyman AE. Incomplete mitral leaflet closure in patients with papillary muscle dysfunction. Circulation. 1981;63:565-71.

5. Timek TA, Lai DT, Tibayan F, Liang D, Daughters GT, Dagum P, et al. Ischemia in three left ventricular regions: insights into the pathogenesis of acute ischemic mitral regurgitation. J Thorac Cardiovasc Surg. 2003;125:559-69.

6. Acker MA, Parides MK, Perrault LP, Moskowitz AJ, Gelijns AC, Voisine P, et al. Mitral-valve repair versus replacement for severe ischemic mitral regurgitation. $N$ Engl J Med. 2014;370:23-32

7. Goldstein D, Moskowitz AJ, Gelijns AC, et al. Two-year outcomes of surgical treatment of severe ischemic mitral regurgitation. N Engl J Med. 2016;374: 344-53.

8. Salmasi MY, Acharya M, Humayun N, Baskaran D, Hubbard S, Vohra H. Is valve repair preferable to valve replacement in ischaemic mitral regurgitation? A systematic review and meta-analysis. Eur J Cardiothorac Surg. 2016;50: $17-28$

9. Virk SA, Sriravindrarajah A, Dunn D, Liou K, Wolfenden H, Tan G, et al. A meta-analysis of mitral valve repair versus replacement for ischemic mitral regurgitation. Ann Cardiothorac Surg. 2015;4:400-10.

10. Mihos CG, Santana O. Mitral valve repair for ischemic mitral regurgitation: lessons from the Cardiothoracic Surgical Trials Network randomized study. J Thorac Dis. 2016;8:E94-9.

11. Lee AP, Acker M, Kubo SH, Bolling SF, Park SW, Bruce CJ, et al. Mechanisms of recurrent functional mitral regurgitation after mitral valve repair in nonischemic dilated cardiomyopathy: importance of distal anterior leaflet tethering. Circulation. 2009;119:2606-14.

12. Ciarka A, Braun J, Delgado V, Versteegh M, Boersma E, Klautz R, et al. Predictors of mitral regurgitation recurrence in patients with heart failure undergoing mitral valve annuloplasty. Am J Cardiol. 2010;106:395-401. 
13. Gelsomino S, van Garsse L, Luca F, Lorusso R, Cheriex E, Rao CM, et al. Impact of preoperative anterior leaflet tethering on the recurrence of ischemic mitral regurgitation and the lack of left ventricular reverse remodeling after restrictive annuloplasty. J Am Soc Echocardiogr. 2011;24:1365-75.

14. Roshanali F, Mandegar MH, Yousefnia MA, Rayatzadeh H, Alaeddini F. A prospective study of predicting factors in ischemic mitral regurgitation recurrence after ring annuloplasty. Ann Thorac Surg. 2007;84:745-9.

15. Gelsomino S, Lorusso R, De Cicco G, Capecchi I, Rostagno C, Caciolli S, et al. Five-year echocardiographic results of combined undersized mitral ring annuloplasty and coronary artery bypass grafting for chronic ischaemic mitral regurgitation. Eur Heart J. 2008;29:231-40.

16. Onorati F, Rubino AS, Marturano D, Pasceri E, Santarpino G, Zinzi S, et al. Midterm clinical and echocardiographic results and predictors of mitral regurgitation recurrence following restrictive annuloplasty for ischemic cardiomyopathy. J Thorac Cardiovasc Surg. 2009;138:654-62.

17. Braun J, Bax JJ, Versteegh MI, Voigt PG, Holman ER, Klautz RJ, et al. Preoperative left ventricular dimensions predict reverse remodeling following restrictive mitral annuloplasty in ischemic mitral regurgitation. Eur J Cardiothorac Surg. 2005;27:847-53.

18. Kron IL, Hung J, Overbey JR, Bouchard D, Gelijns AC, Moskowitz AJ, et al. Predicting recurrent mitral regurgitation after mitral valve repair for severe ischemic mitral regurgitation. J Thorac Cardiovasc Surg. 2015;149:752-61.

19. Valuckiene Z, Ovsianas J, Ablonskyte-Dudoniene R, Mizariene V, Melinyte K, Jurkevicius R. Left ventricular mechanics in functional ischemic mitral regurgitation in acute inferoposterior myocardial infarction. Echocardiography. 2016;33:1131-42.

20. Kleijn SA, Brouwer WP, Aly MF, Russel IK, de Roest GJ, Beek AM, et al. Comparison between three-dimensional speckle-tracking echocardiography and cardiac magnetic resonance imaging for quantification of left ventricular volumes and function. Eur Heart J Cardiovasc Imaging. 2012;13:834-9.

21. Henn MC, Cupps BP, Kar J, Kulshrestha K, Koerner D, Braverman AC, et al. Quantifying "normalized" regional left ventricular contractile function in ischemic coronary artery disease. J Thorac Cardiovasc Surg. 2015;150:240-6.

22. Axel L, Dougherty L. Heart wall motion: improved method of spatial modulation of magnetization for MR imaging. Radiology. 1989;172:349-50.

23. Axel L, Dougherty L. MR imaging of motion with spatial modulation of magnetization. Radiology. 1989;171:841-5.

24. Moulton MJ, Creswell LL, Downing SW, Actis RL, Szabo BA, Vannier MW, et al. Spline surface interpolation for calculating 3-D ventricular strains from MRI tissue tagging. Am J Physiol. 1996;270:H281-97.

25. Moustakidis P, Cupps BP, Pomerantz BJ, Scheri RP, Maniar HM, Kates AM, et al. Noninvasive, quantitative assessment of left ventricular function in ischemic cardiomyopathy. J Surg Res. 2004;116:187-96.

26. Pirolo JS, Creswell LL, Bresina SJ, Perman WH, Szabo BA, Myers KW, et al. Regional myocardial stress distribution from magnetic resonance image-based mathematical models. Ann Thorac Surg. 1991;52:276-84.

27. Pirolo JS, Bresina SJ, Creswell LL, Myers KW, Szabo BA, Vannier MW, et al. Mathematical three-dimensional solid modeling of biventricular geometry. Ann Biomed Eng. 1993;21:199-219.

28. Kar J, Knutsen AK, Cupps BP, Zhong X, Pasque MK. Three-dimensional regional strain computation method with displacement encoding with stimulated echoes (DENSE) in non-ischemic, non-valvular dilated cardiomyopathy patients and healthy subjects validated by tagged MRI. J Magn Reson Imaging. 2015;41:386-96.

29. Kar J, Knutsen AK, Cupps BP, Pasque MK. A validation of two-dimensional in vivo regional strain computed from displacement encoding with stimulated echoes (DENSE), in reference to tagged magnetic resonance imaging and studies in repeatability. Ann Biomed Eng. 2014;42:541-54.

30. Cupps BP, Taggar AK, Reynolds LM, Lawton JS, Pasque MK. Regional myocardial contractile function: multiparametric strain mapping. Interact Cardiovasc Thorac Surg. 2010;10:953-7.

31. Cupps BP, Bree DR, Wollmuth JR, Howells AC, Voeller RK, Rogers JG, et al. Myocardial viability mapping by magnetic resonance-based multiparametric systolic strain analysis. Ann Thorac Surg. 2008;86:1546-53.

32. Lorusso R, Gelsomino S, Vizzardi E, D'Aloia A, De Cicco G, Luca F, et al. Mitral valve repair or replacement for ischemic mitral regurgitation? The Italian Study on the Treatment of Ischemic Mitral Regurgitation (ISTIMIR). J Thorac Cardiovasc Surg. 2013;145:128-39.

33. Gelsomino S, Lorusso R, Capecchi I, Rostagno C, Romagnoli S, Bille G, et al. Left ventricular reverse remodeling after undersized mitral ring annuloplasty in patients with ischemic regurgitation. Ann Thorac Surg. 2008;85:1319-30.
34. Brady BD, Knutsen AK, Ma N, Gardner R, Taggar AK, Cupps BP, et al. MRIbased multiparametric strain analysis predicts contractile recovery after aortic valve replacement for aortic insufficiency. J Card Surg. 2012;27:415-22.

Key Words: ischemic mitral regurgitation, myocardial mechanics, regional contractile injury, strain mapping

\section{Discussion}

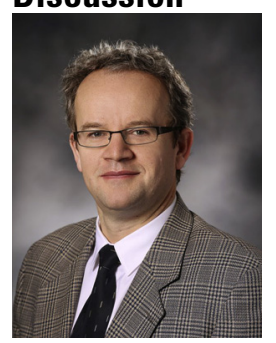

Dr Tomasz Timek (Grand Rapids, Mich). Congratulations on an excellent study and equally excellent presentation. Ischemic mitral regurgitation (MR) continues to frustrate us, and although we now have 2 randomized trials, I do not think we are any closer to a clear answer on how we are supposed to treat these patients.

The key finding of your study is the difference in strain relationships in the myocardial segments that subtend the papillary muscles. This has previously been shown in both animal and clinical work with echocardiography.

My first question relates to the preoperative echo. What was the nature of the preoperative MR jet in patients who had MR and did you see any distinct myocardial regional wall abnormalities in those patients?

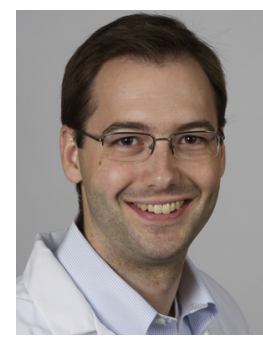

Dr Lancaster. All the patients were patients who were evaluated for surgery and were referred to us with routine clinical echocardiograms. All the patients had moderate (ie, 2+) or greater MR. In terms of the specific characterization of the MR jets, we did not analyze that in this study or compare the regional wall abnormalities by echocardiography with the findings by MR.

Dr Timek. Could you theoretically pick up these differences with echocardiography?

Dr Lancaster. We did not compare these specific patients, but in previous studies we compared magnetic resonance imaging (MRI)-based strain mapping with other methodologies for assessment of regional contractile function and found a very good correlation, for example, with Q waves on electrocardiogram or regional echocardiographic abnormalities. So I expect that we would also see a strong correlation between echocardiography and MR for the patients in this study.

Dr Timek. So this gets into my next question. You showed a $z$ score of almost 2 between the patients who had regurgitation and those who did not, in the subpapillary regions, and, as you mentioned, that essentially means nonviability. Could you draw similar conclusions by simply 
doing a clinical MRI study for viability or a nuclear study? What is the incremental advantage of your technology?

Dr Lancaster. The big advantages of our technology over other methods for viability assessment are the objective and quantitative nature as well as the ability to produce very high resolution visual displays of the data. As I am sure you are aware, other techniques like positron emission tomography and single-photon emission computed tomography as well as dobutamine echocardiography provide qualitative assessment of viability, which is dependent on the observer, and also provide lower resolution and poorer transmural assessment of viability. All of those things are provided by MRI-based strain mapping in better detail.

Other methods for assessing viability by MRI include dobutamine MRI and delayed gadolinium enhancement. Those also are more subjective and less quantitative than this method. So the objectivity and the quantitativeness are the real advantage.

Dr Timek. Your study elegantly confirms, as I already mentioned, the now accumulating and vast evidence that in ischemic MR, the central lesion is really in the subpapillary region. So looking into the future, how does this help us identify the patients who should have mitral replacement, mitral repair, or-for those with moderate MR - just coronary bypass grafting?

Dr Lancaster. As you alluded to, in the recent Cardiothoracic Surgical Trials Network randomized trials, almost $60 \%$ of patients who underwent mitral repair failed by 2 years of follow-up. An interesting conclusion from those studies is when you look at the patients who had repair but did not recur, there were substantial reductions in left ventricular end systolic volume index, demonstrating that they had contractile recovery. The patients who either had mitral valve replacement or had mitral valve repair and recurrence had less evidence of ventricular remodeling.

The key going forward is the ability to delineate viability, especially in the posterior wall and in relation to the posteromedial papillary muscles. That is the real strength of this analysis. We have demonstrated in the past its ability to assess myocardial viability and contractile recovery after surgical intervention, and the ability to precisely localize that to the regions that contribute to ischemic MR I think will help. The direction that we are going is to use this tool to predict the likelihood of success after mitral valve repair.

Dr Timek. Thank you. 
TABLE E1. Circumferential and longitudinal strain data for the ischemic mitral regurgitation (MR) and no-MR groups

\begin{tabular}{|c|c|c|c|}
\hline & Base & Midventricle & Apex \\
\hline \multicolumn{4}{|c|}{ Regional circumferential strain in MR patients $(\mathrm{n}=10)$} \\
\hline Posteroseptal & $-0.11 \pm 0.06$ & $-0.12 \pm 0.05$ & $-0.13 \pm 0.06$ \\
\hline Anteroseptal & $-0.11 \pm 0.06$ & $-0.13 \pm 0.06$ & $-0.14 \pm 0.05$ \\
\hline Anterior & $-0.12 \pm 0.06$ & $-0.15 \pm 0.06$ & $-0.14 \pm 0.05$ \\
\hline Anterolateral & $-0.13 \pm 0.06$ & $-0.15 \pm 0.07$ & $-0.15 \pm 0.06$ \\
\hline Posterolateral & $-0.11 \pm 0.05$ & $-0.13 \pm 0.07$ & $-0.14 \pm 0.09$ \\
\hline Posterior & $-0.07 \pm 0.04$ & $-0.10 \pm 0.06$ & $-0.12 \pm 0.06$ \\
\hline \multicolumn{4}{|c|}{ Regional longitudinal strain in MR patients $(\mathrm{n}=10)$} \\
\hline Posteroseptal & $-0.07 \pm 0.05$ & $-0.08 \pm 0.06$ & $-0.12 \pm 0.05$ \\
\hline Anteroseptal & $-0.06 \pm 0.04$ & $-0.09 \pm 0.05$ & $-0.13 \pm 0.05$ \\
\hline Anterior & $-0.06 \pm 0.04$ & $-0.07 \pm 0.05$ & $-0.11 \pm 0.06$ \\
\hline Anterolateral & $-0.07 \pm 0.05$ & $-0.06 \pm 0.05$ & $-0.08 \pm 0.03$ \\
\hline Posterolateral & $-0.06 \pm 0.04$ & $-0.04 \pm 0.05$ & $-0.10 \pm 0.09$ \\
\hline Posterior & $-0.07 \pm 0.05$ & $-0.04 \pm 0.07$ & $-0.12 \pm 0.09$ \\
\hline \multicolumn{4}{|c|}{ Regional circumferential strain in patients without MR $(n=36)$} \\
\hline Posteroseptal & $-0.12 \pm 0.05$ & $-0.13 \pm 0.06$ & $-0.12 \pm 0.07$ \\
\hline Anteroseptal & $-0.11 \pm 0.05$ & $-0.13 \pm 0.06$ & $-0.13 \pm 0.07$ \\
\hline Anterior & $-0.13 \pm 0.05$ & $-0.15 \pm 0.06$ & $-0.15 \pm 0.06$ \\
\hline Anterolateral & $-0.15 \pm 0.05$ & $-0.18 \pm 0.05$ & $-0.19 \pm 0.06$ \\
\hline Posterolateral & $-0.14 \pm 0.05$ & $-0.15 \pm 0.06$ & $-0.15 \pm 0.07$ \\
\hline Posterior & $-0.10 \pm 0.05$ & $-0.11 \pm 0.05$ & $-0.12 \pm 0.06$ \\
\hline \multicolumn{4}{|c|}{ Regional longitudinal strain in patients without MR $(n=36)$} \\
\hline Posteroseptal & $-0.07 \pm 0.05$ & $-0.10 \pm 0.05$ & $-0.11 \pm 0.06$ \\
\hline Anteroseptal & $-0.05 \pm 0.04$ & $-0.10 \pm 0.05$ & $-0.10 \pm 0.06$ \\
\hline Anterior & $-0.07 \pm 0.04$ & $-0.09 \pm 0.04$ & $-0.10 \pm 0.07$ \\
\hline Anterolateral & $-0.10 \pm 0.05$ & $-0.08 \pm 0.04$ & $-0.10 \pm 0.08$ \\
\hline Posterolateral & $-0.12 \pm 0.06$ & $-0.09 \pm 0.04$ & $-0.13 \pm 0.06$ \\
\hline Posterior & $-0.11 \pm 0.06$ & $-0.10 \pm 0.05$ & $-0.12 \pm 0.06$ \\
\hline
\end{tabular}

